\title{
Häusliche Pflege und die Rolle(n) der Angehörigen
}

\author{
Angelika Feichtner
}

Pflegende und betreuende Angehörige von chronisch oder schwer kranken Patientinnen und Patienten sind mit vielen Herausforderungen konfrontiert und ihre Rolle beinhaltet ein ganzes Bündel von Rollenbildern und Erwartungen. Damit ist diese Rolle nicht so eindeutig, wie es auf den ersten Blick scheint. Durch eine fortschreitende Erkrankung verändern sich die Beziehungen und auch die Rollen innerhalb der Familie (Stenberg et al. 2012). Die unterschiedlichen Erwartungen können zu Rollenkonflikten führen. Besonders deutlich wird das zum Beispiel in der Betreuung betagter Eltern, wenn die Eltern-Kind-Rollen unklar werden.

Eine mögliche Definition von ,Angehörige“ lautet: „Angehörige oder Zugehörige sind all jene Personen, die sich in einer vertrauten, häufig auch verpflichtenden Nähe zum Patienten, zur Patientin befinden. Neben Familienangehörigen können das auch Freunde, Lebensgefährten, Nachbarn oder Kollegen sein" (George et al. 2003, 16). Den Begriff Familie definieren Wright und Leahey $(1994,40)$ so: „The family is who they say they are."

Pflegende Angehörige sind all jene - Angehörige, Freunde und Bezugspersonen -, die eine ihnen nahestehende Person in deren häuslicher Umgebung unentgeltlich pflegen und betreuen. Sie sind also diejenigen, die einen signifikanten, nicht-professionellen oder unbezahlten Beitrag zur Begleitung eines Patienten während fortgeschrittener Erkrankung und der Sterbephase leisten (Payne et al. 2010). Die häusliche Pflege und Betreuung eines schwer kranken Familienmitgliedes kann eine zutiefst bereichernde, wertvolle und sinnstiftende Erfahrung für die gesamte Familie bedeuten. Aber zugleich ist sie auch mit beträchtlichen Herausforderungen und Belastungen für die pflegenden Angehörigen verbunden. Die gemeinsam getragene Fürsorge für den Patienten oder die Patientin kann den Familienzusammenhalt stärken und dadurch, dass die betreuenden Angehörigen in vielfältiger Weise zum Wohlbefinden des Kranken beitragen können, erfahren sie das Gefühl gebraucht zu werden. Damit reduziert sich die Wahrnehmung von Hilflosigkeit, Ohnmacht und Schuld. Die Angehörigen und Bezugspersonen können eine besondere Nähe zum Erkrankten erfahren und trotz hoher Belastung besteht auch für sie die Mög- 
lichkeit des persönlichen Wachsens in der Zeit der Pflege. Die Verbundenheit mit dem erkrankten Familienmitglied nimmt zu und „das gegenseitige Erfahren von großer Liebe ist oft zu beobachten“, wie Bausewein (2005, 68) beschreibt. Laut Statistik Austria sterben in Österreich pro Jahr über 80.000 Menschen $\left(2017\right.$ waren es 83.270 ${ }^{1}$ - die meisten von ihnen nach einer längeren chronischen oder terminalen Erkrankung, verbunden mit Einschränkungen der Lebensqualität, mit Pflegebedürftigkeit und mit tiefgehenden Leiderfahrungen. Laut der Interessengemeinschaft pflegender Angehöriger werden in Österreich derzeit 83 Prozent aller pflegebedürftigen Menschen zuhause betreut. ${ }^{2}$ Nach einer aktuellen Studie zur Angehörigenpflege in Österreich kann von mehr als 801.000 pflegenden Angehörigen ausgegangen werden (Nagl-Cupal et al. 2018). Hinzu kommen mehr als 42.000 Kinder und Jugendliche im Alter von 5 bis 15 Jahren, die ihre kranken Angehörigen pflegen (Nagl-Cupal et al. 2015, 442). Bemerkenswert ist, dass 45 Prozent aller Hauptpflegepersonen älter als 60 Jahre sind und 22 Prozent von ihnen sind älter als 70 Jahre, wie eine Studie von Pochobradsky et al. (2005) zeigt. Viele pflegende Angehörige sind also selbst schon betagt. Die statistischen Erhebungen belegen auch, dass derzeit 78 Prozent der pflegenden Angehörigen keine professionellen Dienste in Anspruch nehmen (Bundesministerium für Arbeit, Soziales und Konsumentenschutz 2016). Das macht deutlich, dass Angehörige die Hauptlast der häuslichen Pflege weitgehend alleine tragen. Dabei sind die Angehörigen gefordert, verschiedene, teilweise einander widersprechende Rollen zu erfüllen.

\section{Angehörige als Haupt-Pflegepersonen}

Pflegebedürftig und auf die Hilfe und Unterstützung anderer angewiesen zu sein, bedeutet immer eine Zäsur und eine gravierende Veränderung des bisherigen Lebens. Dies gilt nicht nur für die Patientinnen und Patienten, sondern ebenso für ihre Angehörigen. Auch ihr Leben verändert sich grundlegend und ganz ähnlich wie die zu Pflegenden müssen auch die betreuenden Angehörigen das, was Normalität für sie bedeutet, neu definie-

1 Vgl. Statistik Austria: Statistik der natürlichen Bevölkerungsbewegung, in: http://w ww.statistik.at/webde/statistiken/menschen_und_gesellschaft/bevoelkerung/gestor bene/index.html [18.05.2018].

2 Vgl. Interessengemeinschaft pflegender Angehöriger: FACTSHEET - Pflegesituation in Österreich, in: https://www.ig-pflege.at/downloads/news/2017/FACTSHEET_ 2017_LOGO.pdf?m=1495009759 [18.02.2020]. 
ren (Carlander et al. 2006). Sie sind gefordert, ihren Alltag immer mehr an die Bedürfnisse des Kranken anzupassen, und zunehmend müssen sie nicht nur die basale Versorgung, sondern auch komplexe Pflege- und Betreuungsaufgaben übernehmen. Häufig müssen sie dabei eigene Schamund Ekelgrenzen überschreiten. Sie erfüllen die Pflege-Aufgaben, ohne dafür entsprechend ausgebildet und vorbereitet zu sein, und die emotionale Nähe zum Kranken erweist sich dabei oft als zusätzlicher Belastungsfaktor. Die Praxis zeigt, dass Angehörige grundsätzlich eine hohe Bereitschaft haben, die Verantwortung für die häusliche Pflege zu übernehmen, zugleich ist aber deutlich, dass sie die Tragweite der Entscheidung zur Übernahme der Betreuung oft nicht realistisch einschätzen können. Viele Angehörige übernehmen die Fürsorge aufgrund von Bindung, Loyalität und Solidarität. Manche Angehörige betrachten ihre Bereitschaft zur Übernahme der häuslichen Fürsorge als moralische Pflicht oder auch schlicht als Selbstverständlichkeit. Möglicherweise sind aber auch früher gegebene Versprechen oder erlebte negative Erfahrungen mit institutioneller Versorgung ausschlaggebend dafür, dass Angehörige die häusliche Betreuung übernehmen (Stajduhar und Davies 2005). Mitunter sind auch Schuldgefühle dem Patienten oder der Patientin gegenüber, finanzielle Aspekte oder der Erwartungsdruck des familiären Umfeldes entscheidende Faktoren für die Übernahme der Pflege und Betreuung. Damit scheint die Übernahme dieser Verantwortung oft weniger die Konsequenz eines aktiven Entscheidungsprozesses zu sein, sondern ein eher unreflektiertes Erfüllen von Erwartungen (Kunstmann 2010, 486). Häufig sind es zunächst kleine, als selbstverständlich wahrgenommene, unterstützende Maßnahmen, die Angehörige leisten, aber mit dem Fortschreiten der Erkrankung steigt der Pflegebedarf kontinuierlich. Der Übergang von der üblichen, alltäglichen Fürsorge zur Pflege verläuft oft allmählich und viele Angehörige nehmen sich selbst zunächst gar nicht bewusst in der Rolle als Pflegende wahr. So geraten sie mitunter, unabhängig von ihrer Eignung oder ihrem Wunsch, allmählich oder auch plötzlich in die Rolle der Haupt-Pflegeperson. Die Dauer der häuslichen Pflegesituation ist ebenso wie ihr Verlauf hinsichtlich der Intensität der Pflege meist nicht abschätzbar. Pflegende Angehörige befinden sich daher permanent in einer von Unsicherheit geprägten Situation. Das mögliche Auftreten von krisenhaften Situationen verstärkt die Wahrnehmung von genereller Unsicherheit. In Anlehnung an die Bedürfnispyramide nach Maslow (Zalenski und Raspa 2006) gilt das Erleben von Sicherheit aber als ganz wesentlicher Faktor für Lebensqualität.

Fast immer sind es die Frauen in der Familie, die, oft zunächst vorübergehend, Pflege- und Betreuungsverantwortung übernehmen. Gröning begründet das damit, „dass sich Frauen aufgrund der jetzigen gesellschaftli- 
chen Bedingungen und vor dem Hintergrund der geschlechtsspezifischen Sozialisation dem normativen Anspruch zur Pflegeübernahme einfach schlechter entziehen können als Männer" (Gröning et al. 2004, 51). Auch bei den pflegenden Kindern und Jugendlichen sind fast 70 Prozent $(69,8)$ weiblich (Nagl-Cupal et al. 2015). Vor allem für berufstätige Frauen bedeutet die Übernahme der häuslichen Betreuung eines chronisch oder schwer kranken Angehörigen eine enorme zusätzliche Belastung. Nimmt der Pflegebedarf zu, sehen sich die Frauen aufgrund fehlender Vereinbarkeit von Betreuungsarbeit und Beruf meist dazu gezwungen, ihre Erwerbstätigkeit aufzugeben - mit entsprechend nachteiligen Folgen für ihre berufliche Biografie. Pflegende Angehörige fühlen sich offenbar auch einem gewissen Leistungsdruck ausgesetzt und interpretieren es als persönliches Versagen, wenn ihnen die Vereinbarkeit von Erwerbs- und Pflegearbeit nicht gelingt (Arksey 2002).

\section{Angehörige als Mitbetroffene}

Studien zeigen, dass Frauen, die häusliche Pflegearbeit leisten, einem wesentlich höheren Burnout-Risiko ausgesetzt sind als Männer in ähnlicher Situation und sie erhalten auch deutlich weniger Unterstützung aus ihrem Umfeld als männliche Betreuer (Payne 2013). Während einem pflegenden Mann nicht nur gesellschaftliche Bewunderung und Wertschätzung, sondern auch praktische Unterstützung sicher ist, gilt es bei Frauen nahezu als selbstverständlich, dass sie die häusliche Pflege eines schwerkranken Familienmitgliedes leisten.

Ob eine häusliche Betreuung generell möglich ist und ob dem Wunsch vieler Patienten und Patientinnen, bis zum Versterben zu Hause bleiben zu können, entsprochen werden kann, hängt vor allem davon ab, was die Angehörigen leisten können (Gomez und Higginson 2006). Damit sind Angehörige von chronisch und schwer Kranken einem mehrfachen Druck ausgesetzt. In der Praxis ist zu beobachten, dass das Zuhause-Bleiben-biszuletzt oft idealisiert wird und pflegende Angehörige daher trotz komplexer Belastungen weit über die Grenze des Leistbaren gehen. Selbst massiv belasteten Angehörigen fällt es oft enorm schwer, sich aus einmal übernommenen Pflegeaufgaben wieder zurückzuziehen. Häufig fällt es ihnen aber auch schon schwer, Unterstützung durch einen professionellen Pflegedienst anzunehmen. Die Gründe dafür sind vielschichtig. Sie reichen von der Haltung „Das müssen wir alleine schaffen“ oder „Ich habe es versprochen" bis hin zu finanziellen Gründen, die verhindern, dass pflegende Angehörige rechtzeitig professionelle Unterstützung suchen. Also wird un- 
ter zunehmend schwierigen Bedingungen die häusliche Betreuung aufrechterhalten - oft um einen hohen Preis. Dies hat natürlich auch Auswirkungen auf das Wohlbefinden der Patientinnen und Patienten, die mit ihren pflegenden Angehörigen ein aufeinander bezogenes und wechselseitig interagierendes System bilden. Es ist nachvollziehbar, dass überlastete und überforderte Angehörige kaum dazu in der Lage sein werden, eine adäquate, Sicherheit und Geborgenheit vermittelnde Betreuung zu bieten. Pflegende Angehörige tragen die gesamte Verantwortung (Brobäck und Berterö 2003), viele sind 24 Stunden für den Patienten oder die Patientin zuständig, und das 7 Tage in der Woche, über Monate und manchmal auch über Jahre. Oft haben sie kaum Rückzugs- und Erholungsmöglichkeiten, sie fühlen sich tatsächlich alleine verantwortlich und als ,immer im Dienst" (George et al. 2003). Die ständige Sorge um einen kranken Menschen kann bei pflegenden Angehörigen zu permanenter Anspannung sowie zu Unsicherheit und Ängsten führen. Viele pflegenden Angehörige erleben ein für sie schier unerträgliches Ausmaß des Gebraucht-Werdens und leiden unter dem permanenten Gefühl des Angebunden-Seins.

Was ich dringend brauche, ist Ruhe. Ich müsste mich körperlich und auch emotional ausruben können. Aber ich finde keine Rube, nicht wenn ich bei ihm bin, und erst recht nicht, wenn ich nicht bei ihm bin. Deshalb kann ich auch nicht aus dem Haus gehen. (Aussage einer pflegenden Angehörigen)

Durch das Gefühl, immer präsent sein zu müssen, werden die sozialen Kontakte stark eingeschränkt und die Gefahr der Isolation droht (Stenberg et al. 2012). Diese Einsamkeit, verbunden mit der Wahrnehmung anhaltender Überforderung und hoher emotionaler Belastung, kann zu einem Verlust der Empathie-Fähigkeit führen und nicht selten ist dies auch der Wegbereiter für Gewalt. Auch Stajduhar (2013) beschreibt die soziale Isolation unter pflegenden Angehörigen als ein weit verbreitetes Phänomen. Sie verweist auch darauf, dass Angehörige sich selbst gar nicht als legitime Empfänger von Hilfe betrachten und eine mögliche Unterstützung oft auch daran scheitert. In einer Situation, in der die pflegenden Angehörigen der Unterstützung und Hilfe bedürfen, erfahren sie Isolation und Einsamkeit. (Feichtner/Pußwald 2017, 176). Und selbst dann, wenn Unterstützung, etwa in Form eines ehrenamtlichen Präsenzdienstes oder eines Hauskrankenpflege-Dienstes, konkret angeboten wird, fällt es manchen Angehörigen aus den genannten Gründen überaus schwer, diese entlastenden Angebote anzunehmen. Im hohen Anspruch der Angehörigen, die häusliche Pflege alleine zu bewältigen und rund um die Uhr verfügbar und präsent zu sein, sehen Kreyer und Pleschberger (2014) aber auch eine Strate- 
gie, die Angehörigen hilft, die Situation unter Kontrolle zu behalten. Häusliche Pflege ist von wechselnden Anforderungen und Unsicherheiten geprägt und das Bedürfnis Angehöriger nach Kontrolle kann daher auch als ein Versuch der Entlastung betrachtet werden. Wird die Unterstützung durch ein professionelles Hauskrankenpflege-Team angenommen, erfahren die Angehörigen dies oft wie eine sichere Basis, die das Gefühl von Kontrolle über die oft als bedrohlich empfundene Situation vermitteln kann.

\section{Angehörige als Sekundärpatientinnen und-patienten}

Wenn die Tage und zunehmend auch die Nächte von den Bedürfnissen des Kranken bestimmt sind und Angehörige kaum oder gar keine Möglichkeiten einer Auszeit haben, können die körperlichen, emotionalen und psychischen Belastungen durch die Betreuungsaufgabe dazu führen, dass sie selbst zu Patientinnen und Patienten werden (Stajduhar et al. 2010). Kristjanson und Aoun (2004) bezeichnen Angehörige daher auch als „hidden patients“, als Patientinnen und Patienten zweiter Ordnung, denn sie benötigen oft ähnlich wie die Patientinnen und Patienten Unterstützung und Hilfe. Erschöpfung, Ängste, Schlafstörungen, Depressionen und anhaltende Niedergeschlagenheit sind häufige Reaktionen Angehöriger auf die Belastungen in der häuslichen Pflege.

„Caregiver Burden bezeichnet das Ausmaß, in dem die Angehörigen durch die (häusliche) Pflege negative Auswirkungen in sozialer, finanzieller, körperlicher und spiritueller Hinsicht erfahren." (Zarit et al. 1986, 260)

Diese Belastungen, in der Literatur auch als „Caregiver Burden“ (Zarit et al. 1986) bezeichnet, nehmen mit Fortschreiten der Erkrankung und mit der Intensität der Pflegebedürftigkeit zu. Durch die erhöhte körperliche und emotionale Belastung, die permanente Unsicherheit und die regelmäßige Unterbrechung der Nachtruhe kann es zu beträchtlichen körperlichen und psychischen Erschöpfungszuständen bis hin zu schwerwiegenden Erkrankungen kommen. Zu den besonderen Risikofaktoren und Verstärkern für die Entwicklung eines solchen Erschöpfungssyndroms bei pflegenden Angehörigen zählen: 
- unfreiwillige Übernahme der Pflege- und Betreuungsverantwortung,

- haupt- oder allein verantwortlich zu sein; fehlende professionelle Unterstützung,

- keine oder zu geringe Pausen, zu wenige Zeiten der Ruhe und Distanz,

- mangelnde Selbstfürsorge-Fähigkeit, geringe Aufmerksamkeit für eigene Gesundheit,

- kaum oder keine Sozialkontakte, Isolation und Einsamkeit,

- bestehende Depression, depressive Symptome oder anhaltend gedrückte Stimmung,

- geringe Coping-Strategien; reduzierte Fähigkeit, Hilfe zu suchen und anzunehmen (Stajduhar/Davies 2005),

- Konfrontation mit Leid, Angst vor dem drohenden Verlust,

- ausgeprägte Schuldgefühle; das Gefühl, nicht genug für den Kranken tun zu können

- Störungen im Schlaf-Wach-Rhythmus, Schlafmangel und Schlafstörungen (Adelmann et al. 2014),

- finanzielle Belastungen; Götze et al. (2014) beschreiben einen deutlichen Zusammenhang zwischen Erschöpfungs- und Überlastungssymptomen und finanziellen Belastungen.

Mehrere dieser Risikofaktoren stellen zugleich auch einige der bekannten Folgen eines Erschöpfungssyndroms dar. So können etwa Schlafstörungen oder eine bestehende Depression sowohl Auslöser als auch Resultat eines Caregiver-Burdens sein. Es ist daher bedeutsam, dass professionell begleitende Teams, wie etwa die Hauskrankenpflege, Signale einer drohenden Erschöpfung bei Angehörigen möglichst frühzeitig erfassen. Dafür stehen verschiedene bewährte Assessment-Instrumente wie etwa der Zarit-Burden-Fragebogen (Seng et al. 2010), das Carers' Alert Thermometer (CAT) (Knighting et al. 2015) oder der modifizierte Caregiver Strain Index (Kruithof et al. 2015) zur Verfügung.

\subsection{Warnsignale, die auf eine zunehmende körperliche und seelische Erschöpfung hinweisen}

- Energiemangel, Schwächegefühl und chronische Müdigkeit

- das Gefühl der Überforderung, dass einem alles zu viel ist

- Nervosität und innere Unruhe, Schlafstörungen

- Niedergeschlagenheit, Hoffnungslosigkeit

- Gefühl innerer Leere, Gefühlslosigkeit, Gedanken der Sinnlosigkeit 
- Angstgefühle, Schuldzuweisungen, Gereiztheit, Gefühle von Wut und Ärger

- Vermeiden von Kontakten mit anderen Menschen, sozialer Rückzug

- Magen-Darm-Beschwerden, Herz-Kreislauf-Störungen, anhaltende Kopf- oder Rückenschmerzen

Wenn diese alarmierenden Anzeichen einer drohenden Erschöpfung nicht erkannt werden und nicht entsprechend rasch Entlastung geboten wird, kann sich daraus ein ausgeprägtes Fatigue-Syndrom entwickeln. Eine schon ältere Studie von Jensen und Given (1993) zeigte, dass 53 Prozent der befragten pflegenden Angehörigen unter anhaltender Fatigue leiden. Der Grad dieser Erschöpfung korreliert mit dem Ausmaß, in welchem die Tage und Nächte der Angehörigen durch die Bedürfnisse des Kranken bestimmt sind. Um an den Anforderungen in einer häuslichen Pflege nicht zu zerbrechen und physisch wie psychisch gesund zu bleiben, ist es daher unerlässlich, dass pflegende Angehörige regelmäßige Auszeiten haben und dass sich die Verantwortung für die Versorgung des Kranken auf mehrere Personen aufteilt. Das bedeutet für die einzelnen Betreuungspersonen Entlastung und ein deutlich geringeres Risiko der Überforderung. Ebenso stellen eine frühzeitige psychosoziale und praktische Unterstützung der pflegenden Angehörigen durch professionelle Hauskrankenpflege oder Palliativteams eine wesentliche Form der Entlastung dar.

Wie von Payne (2013) beschrieben, belegen auch Gallicchio et al. (2002), dass Frauen gegenüber Männern ein zweifach erhöhtes Risiko tragen, in derartige Erschöpfungszustände zu geraten. Auch hier liegt der Grund vor allem in den herrschenden traditionellen Rollenbildern. Männer sind, anders als Frauen, keinem sozialen Erwartungsdruck ausgesetzt, die Pflege zu übernehmen. Auch kann grundsätzlich davon ausgegangen werden, dass „Männer die Übernahme einer Pflegeverantwortung eher reflektieren können, während bei Frauen ein verinnerlichtes Gefühl der Verpflichtung dazu besteht, welches weniger Raum für Reflexion zulässt“, wie Langehennig $(2012,22)$ schreibt. Als Konsequenz andauernder Überlastung kann das Erfüllen der Fürsorgepflichten dazu führen, dass die pflegenden Angehörigen selbst erkranken. Sie werden zu Sekundärpatientinnen und -patienten, und nicht selten werden sie in der Folge selbst pflegeund betreuungsbedürftig (Bold/Deußen 2013, 109).

In der Pflege meines Mannes ist nicht nur mein Körper, sondern auch meine Seele kaputt gegangen. (Aussage einer pflegenden Angehörigen) 


\section{Angehörige als Expertinnen und Experten}

In der häuslichen Pflege eignen sich die Angehörigen zunehmend Wissen über die Erkrankung und pflegerisches Können an. Sie werden zu Expertinnen und Experten für die Versorgung ihres Kranken und kennen den Patienten oder die Patientin meist sehr gut, wissen also um seine oder ihre Vorlieben und Abneigungen. Und selbst bei sehr eingeschränkter Kommunikationsfähigkeit des Patienten oder der Patientin können sie deren Wünsche gleichsam erraten. Oft sind es die kleinen, vertrauten und liebevollen Gesten, das Erfüllen spontaner Wünsche und das fürsorgliche Bemühen der Angehörigen, die ganz wesentlich zum Wohlbefinden der Kranken beitragen. Durch das Erlernen pflegerischer Fertigkeiten, die zunehmende Fähigkeit der Angehörigen, Symptome zu erkennen und sie richtig zu interpretieren, und auch durch immer mehr Wissen um Wirkungen und Nebenwirkungen der Medikamente steigt die Kompetenz der pflegenden Angehörigen. Dabei darf jedoch nicht übersehen werden, dass sie sich einerseits kompetent, andererseits oft zugleich auch sehr hilflos fühlen. Häufig werden Gefühle von Verunsicherung und Unzulänglichkeit beschrieben. Abhängig vom Ausmaß und der Qualität professioneller Unterstützung lernen die Angehörigen in der häuslichen Pflege oft nur mittels Versuch und Irrtum. Auch wenn sie damit oft erfolgreich sind, so ist dies immer auch mit einem hohen $\mathrm{Ma}$ an Unsicherheit verbunden. Pflegende Angehörige äußern häufig Ängste, etwas falsch zu machen (Stajduhar 2013, 659). Es ist daher besonders wichtig, dass pflegende Angehörige auf fachliche Anleitung und Schulung in pflegerischen Belangen und auf die Unterstützung eines Hauskrankenpflege- oder Palliativteams zählen können. Dabei hat sich gezeigt, dass ein rund um die Uhr telefonisch erreichbarer Bereitschaftsdienst eines professionellen Pflegedienstes die subjektive Sicherheit der Angehörigen deutlich erhöht. Es wird als große Entlastung wahrgenommen, wenn sich die Angehörigen in unsicheren Situationen telefonisch rückversichern können. Allein schon das Wissen um diese Möglichkeit wirkt sich positiv auf ihr Sicherheitsgefühl aus.

\section{Angehörige als Bewahrerinnen und Bewahrer der Normalität}

Laut einer Untersuchung von Stajduhar et al. (2010) verbringen Menschen mit terminalem Krankheitsverlauf die meiste Zeit des letzten Lebensjahres zu Hause. Sie werden dort betreut und gepflegt und die Mehrheit wünscht sich auch, dort zu sterben (Kristjanson/Aoun 2004). Dieser Wunsch entspringt meist nicht nur dem Bedürfnis nach Geborgenheit in der vertrau- 
ten Umgebung, er ist auch mit der Sehnsucht nach Normalität verbunden. $\mathrm{Zu}$ Hause können, anders als bei institutioneller Betreuung, individuelle Tagesabläufe und persönliche Rituale, auch in fortgeschrittener Krankheitssituation, weitgehend aufrechterhalten werden. Für die Angehörigen kann die Aufrechterhaltung des familiären Alltags neben den Pflege- und Betreuungserfordernissen aber eine große Herausforderung darstellen. Sie geraten mitunter in ein Spannungsfeld zwischen ihren eigenen Bedürfnissen, den familiären Anforderungen, den Bedürfnissen des Kranken und dem Bemühen um Normalität. Pflegende Angehörige beschreiben es gleichsam als Balanceakt, als „living on the edge“ (Northfield/Nebauer 2010, 571), den unterschiedlichen Bedürfnissen gerecht zu werden. Gelingt es Familien, Normalität (zumindest einigermaßen) wiederherzustellen, so kann trotz dynamischer Veränderungen des Krankheits- oder Betreuungsgeschehens für eine gewisse Zeit Stabilität und Kontrolle über die Lebenssituation und deren Anforderungen erlebt werden (Penrod et al. 2012). Diese relative Sicherheit und Stabilität der Angehörigen wirkt sich ganz unmittelbar positiv und beruhigend auf die Patientinnen und Patienten aus und oft ist zu beobachten, wie sich auch ihre Situation stabilisiert.

\section{Angehörige als Bündnispartnerinnen und-partner für professionell Betreuende}

Für die Kranken stellen ihre Angehörigen die bedeutsamste Unterstützung dar, daher sind sie auch von den professionell Betreuenden als wichtige Bezugspersonen und Partnerinnen oder Partner anzuerkennen (Stenberg et al. 2012). In der häuslichen Betreuung gewährleisten Angehörige die Kontinuität der Pflege, sie leisten nicht nur Basispflege, sondern meist auch komplexe pflegerische Versorgung. Sie verabreichen Medikamente, überwachen die Symptomlinderung und stellen auch eine überaus wichtige Informationsquelle für das professionelle Betreuungsteam dar. Angehörige beschaffen Pflegehilfsmittel und Medikamente, stellen Anträge und organisieren Arztvisiten und Kontrolltermine, was, wie Penrod et al. $(2012,7)$ beschreiben, mitunter zu „hektischen Stundenplänen“ der Angehörigen führt. Pflegende Angehörige berichten oft, dass diese organisatorischen Aufgaben eine große Belastung darstellen.

Angehörige stellen eine wertvolle und entlastende Ressource nicht nur für die Kranken, sondern auch für das professionell betreuende Team dar. Es ist daher von besonderer Bedeutung, dass den Angehörigen mit Wertschätzung und Respekt begegnet wird. Mit Fortschreiten der Erkrankung sind pflegende Angehörige bisweilen mit rasch wechselnden Anforderun- 
gen konfrontiert. Krisenhafte Situationen sind nicht selten. Eine mit den Angehörigen gemeinsam erstellte vorausschauende Planung für Krisenund Notfallsituationen trägt dazu bei, dass die Angehörigen auch in Akutsituationen handlungsfähig bleiben und diese Krisen durch entsprechende, vorab angeordnete Maßnahmen bewältigen können (Lederer et al. 2015). Von professionell Betreuenden brauchen pflegende Angehörige nicht nur umfassende Information und Schulung, sondern auch Wertschätzung und Anerkennung als Partnerinnen und Partner im gemeinsamen Bemühen um das Wohlergehen der Patientin oder des Patienten. Das Bündnis zwischen Angehörigen und professionell Pflegenden gewährleistet eine fachlich entsprechende Versorgung der Patientinnen und Patienten und auch die bestmögliche Unterstützung der pflegenden Angehörigen. Eine mögliche Überforderung der Angehörigen kann frühzeitig erfasst werden und durch konkrete Entlastungsangebote kann drohender Erschöpfung entgegengewirkt werden.

\section{Angehörige als Trauernde}

Neben den vielfältigen Anforderungen, mit denen pflegende Angehörige konfrontiert sind, darf nicht übersehen werden, dass sie nicht nur Mitbetroffene, sondern zugleich auch Trauernde sind. Sie haben nicht nur die Trauer um den Verlust des bisherigen Lebens zu bewältigen, sie erfahren auch antizipierte Trauer um den drohenden endgültigen Verlust des Patienten oder der Patientin. Eine aktuelle dänische Studie von Nielsen et al. (2016) zeigt, dass bei 15 Prozent der 432 untersuchten pflegenden Angehörigen starke antizipierte Trauersymptome in Form eines Pre-Loss-Syndroms festgestellt wurden. Dabei ist den Betroffenen häufig nicht bewusst, dass die intensiv erlebten Gefühle bereits Ausdruck ihrer Trauer sind. Während bisher davon ausgegangen wurde, dass sich eine antizipierte Trauer positiv auf die Trauer nach dem Tod des Patienten oder der Patientin auswirkt, belegt eine weitere Untersuchung von Nielsen et al. (2017) einen erschwerenden, negativen Einfluss. Ähnlich wie schon Nanni et al. (2014) gehen auch Nielsen et al. davon aus, dass eine starke antizipatorische Trauerreaktion einen Indikator für eine nachfolgende erschwerte Trauer darstellt.

Für die professionelle Unterstützung der betreuenden Angehörigen ist es wichtig, diese Trauerreaktionen zu erkennen und die Angehörigen in beiden Rollen - als mitbetroffene Pflegeperson und als Trauernde - wahrzunehmen (Brobäck/Berterö 2003). Wenn dies gelingt und Angehörige entsprechende Unterstützung durch ein professionelles Team erhalten, 
können die Erfahrungen in der häuslichen Betreuung des Kranken zur Bewältigung der momentanen und auch der nachfolgenden Trauer beitragen.

\section{Angehörige als Leistungserbringerinnen und-erbringer für das Sozial-und Gesundheitssystem}

Es kann davon ausgegangen werden, dass unser Gesundheitssystem ohne die Leistungen pflegender Angehöriger nicht aufrechtzuerhalten wäre. Sie leisten mit der häuslichen Betreuung einen unschätzbar wichtigen Beitrag, oft zulasten ihrer eigenen Gesundheit. Würde auch nur die Hälfte der aktuell 947.000 pflegenden Angehörigen die Betreuung niederlegen, so würde das österreichische Gesundheits- und Pflegesystem vor nicht zu bewältigenden organisatorischen und finanziellen Herausforderungen stehen (Sell 2018). Damit ist auch der ökonomische Wert häuslicher Pflege nicht zu unterschätzen. Den pflegenden Angehörigen gebührt Dank, Respekt, gesellschaftliche Anerkennung und Wertschätzung. Das erklärt im Übrigen auch die frühere Sozialministerin Beate Hartinger-Klein und ergänzt: „Wir werden die Ergebnisse der Studie nun genau analysieren und prüfen bereits erste, kurzfristig umsetzbare Maßnahmen zur Beratung und Sensibilisierung.“ Weiters versichert sie: „Auf Basis der Studienergebnisse werden abschließend Empfehlungen ausgearbeitet, die sowohl für die Lebensqualität und das Wohlbefinden pflegender Angehöriger als auch für die Weiterentwicklung des österreichischen Systems für pflegende Angehörige notwendig sind. ${ }^{\text {"3 }}$

Empfehlungen zur Verbesserung der Situation von häuslicher Pflege sind hinlänglich bekannt. Um Pflege und Betreuung zu Hause längerfristig leisten zu können und dabei auch gesund zu bleiben, brauchen Angehörige nicht weitere Empfehlungen, sondern ganz konkrete, praktische und psychosoziale Unterstützung sowie jede mögliche Form der Entlastung. Im Fokus professionell betreuender Teams kann daher nicht nur der Patient bzw. die Patientin sein, das Bemühen des Teams muss sich auch auf die pflegenden Angehörigen ausweiten - es bedarf einer familienorientierten Betreuung. Die vielfältigen Belastungen, denen pflegende Angehörige ausgesetzt sind, wurden bereits umfassend erforscht, aber darüber,

3 Hartinger-Klein: „Pflege braucht Service und Qualität, in: https://www.ots.at/press eaussendung/OTS_20180511_OTS0024/hartinger-klein-pflege-braucht-service-undqualitaet [18.02.2020]. 
welche entlastenden Maßnahmen von den Angehörigen tatsächlich als wirksam erlebt werden, bedarf es noch weiterer Forschung.

\subsection{Interventionen zur Entlastung pflegender Angehöriger}

Im Rahmen eines Familiengespräches kann geklärt werden, wer aus der Familie und dem näheren sozialen Umfeld neben der Haupt-Pflegeperson mitbetreuen und Unterstützung bieten kann. In diesen Familiengesprächen können auch die individuellen Belastungen und Bedürfnisse, sowohl der Patientinnen und Patienten als auch der Angehörigen erfasst werden. In der Praxis erweisen sich diese Gespräche vielfach als klärend und entlastend.

Pflegende Angehörige bedürfen einer ressourcenorientierten fachlichen Beratung und Begleitung durch ein professionelles Hauskrankenpflegeoder Palliativteam. Wie die bereits eingangs erwähnte Statistik des Bundesministeriums für Arbeit, Soziales und Konsumentenschutz (2016) zeigt, verzichten derzeit 78 Prozent der pflegenden Angehörigen auf professionelle Unterstützung. Es wäre daher zu überlegen, ob eine verpflichtende Einbeziehung eines professionellen Dienstes, vor allem bei höheren Pflegestufen, zur Entlastung beitragen könnte. Dabei wäre eine Personalkontinuität in der professionellen Unterstützung vorteilhaft. Flexible, kurzfristig abrufbare Angebote für stundenweise Präsenzdienste würden einen weiteren Beitrag zur Entlastung der Angehörigen bieten.

Die Gewährleistung regelmäßiger und ausreichender Auszeiten für die Haupt-Pflegeperson durch das soziale Umfeld oder durch ehrenamtliche Präsenzdienste würde entlasten. Diese Unterstützungsangebote würden auch der zunehmenden sozialen Isolation der Angehörigen entgegenwirken. Die Praxis zeigt: Je früher diese Entlastungsstrategien eingesetzt werden, desto eher können sie von den Betroffenen akzeptiert werden. Da sich die Belastungen durch permanent gestörte Nachtruhe besonders negativ auf die Gesundheit pflegender Angehöriger auswirken, wäre die Möglichkeit eines anlassbezogenen, fallweisen Nachtdienstes wünschenswert.

Durch ein systematisches Assessment mit adäquaten Assessment-Instrumenten können die Belastung und eine mögliche Überforderung pflegender Angehöriger erfasst werden. (Honea et al. 2008). Die wiederholte Einschätzung der Belastung pflegender Angehöriger ist wesentlich, um Erschöpfungszuständen im Sinne eines Caregiver-Burdens frühzeitig und wirksam begegnen zu können.

Wiederholte Information, Beratung, praktische Anleitung und Hilfestellung durch professionelle Pflegeteams führen zu Entlastung und einer hö- 
heren Sicherheit pflegender Angehöriger. Bei Fragen oder Unsicherheit bietet die Erreichbarkeit einer telefonischen Pflege-Beratung Entlastung, auch ein pflegerischer 24-Stunden-Bereitschaftsdienst würde dazu beitragen, dass Angehörige die häusliche Pflege längerfristig leisten können.

Vorausschauende Krisen- und Notfallplanung mit klaren schriftlichen Handlungsanleitungen und eine rund um die Uhr erreichbare telefonische Beratung stellen sicher, dass krisenhafte Situationen im häuslichen Umfeld bewältigt werden können und nicht zu einer vermeidbaren Krankenhauseinweisung führen.

Um die finanziellen Belastungen in der häuslichen Pflege zu reduzieren, bedarf es einer Valorisierung des Pflegegeldes und höhere Zuschüsse für vorhandene Dienste und Hilfsmittel.

Da die Vereinbarkeit von Berufstätigkeit und häuslicher Pflege für viele Angehörige ein großes Problem darstellt und die häusliche Betreuung oft an dieser Unvereinbarkeit scheitert, braucht es neue Arbeitszeitmodelle und entsprechende Unterstützung für berufstätige pflegende Angehörige.

\section{Fazit}

Durch die Pflegebedürftigkeit eines Familienmitgliedes ergeben sich für die Angehörigen zusätzlich zur bisherigen Grundbelastung durch Beruf und Alltagsroutine vielfältige Aufgaben, die übernommen werden müssen. Häufig verändern sich die bisherigen Rollenverteilungen innerhalb der Familie und des sozialen Umfeldes. Lang erprobte und eingespielte Rollen und Beziehungen verändern sich und die Angehörigen sind dadurch mit dem Erfordernis einer Neukonstruktion ihrer Rolle und Identität konfrontiert.

Viele Angehörige erleben die häusliche Pflege eines schwer kranken Familienmitgliedes durchaus als zutiefst positive und wertvolle Erfahrung. Dabei darf jedoch nicht übersehen werden, dass die häusliche Pflege und Betreuung eines schwerkranken Menschen eine enorme Herausforderung für das gesamte familiäre System darstellt. Nur wenn alle Mitglieder der Familie eine Betreuungsverpflichtung übernehmen, Unterstützungsangebote professioneller Dienste und auch aus dem Freundeskreis angenommen werden und nur wenn der Hauptpflegeperson auch regelmäßig freie Zeit zur Erholung zugestanden wird, kann die häusliche Pflege zu einer wertvollen und bereichernden Erfahrung werden.

Angehörige leisten oft Pflege und Betreuung rund um die Uhr; sie tun dies, ohne über die entsprechende Ausbildung dafür zu verfügen, und sie leisten diese Arbeit oft bis an die Grenze ihrer Kräfte. In einer Situation, in 
der sie selbst enormen emotionalen und körperlichen Belastungen ausgesetzt sind, stellen sie eigene Bedürfnisse in den Hintergrund. Damit pflegende Angehörige diese Leistung erbringen können und durch die Belastungen nicht selbst erkranken und zu Patientinnen oder Patienten werden, müssen dafür bestmögliche Rahmenbedingungen geschaffen und umfassende Unterstützung geboten werden. Noch mehr als bisher wird es darauf ankommen, dass pflegende Angehörige gesellschaftliche Solidarität und Wertschätzung erfahren - eine Wertschätzung, die sich auch in entsprechenden Rahmenbedingungen für die häusliche Pflege ausdrückt.

\section{Literatur}

Adelman, Ronald D./Tmanova, Lyubov L./Delgado, Diana/Dion, Sarah/Lachs, Mark S.: Caregiver Burden. A Clinical Review, in: Journal of the American Medical Association 311/10 (2014) 1052-1060.

Arksey, Hilary: Combining informal care and work. Supporting carers in the workplace, in: Health and Social Care in the Community 10/3 (2002) 151-161.

Bausewein, Claudia: Sterbende begleiten. Ignatianische Impulse, Würzburg: Echter ${ }^{2} 2009$.

BMASGK veröffentlicht Studie zur Einsicht in die Situation pflegender Angehöriger, in: https://www.ots.at/presseaussendung/OTS_20180816_OTS0108/bmasgkveroeffentlicht-studie-zur-einsicht-in-die-situation-pflegender-angehoeriger [18.02.2020].

Bold, Stefanie/Deußen, Marina: Vereinbarkeit von Beruf und Pflege, München: Hampp 2013 (= Praxisorientierte Personal- und Organisationsforschung 16).

Brobäck, Gunilla/Berterö, Carina: How next of kin experience palliative care of relatives at home, in: European Journal of Cancer Care 12/4 (2003) 339-346.

Bundesministerium für Arbeit, Soziales und Konsumentenschutz (Hg.): Österreichischer Pflegevorsorgebericht 2016, Wien: 2017, in: https://broschuerenservice. sozialministerium.at/Home/Download?publicationId=449 [21.02.2020].

Carlander, Ida/Sahlberg-Blom, Eva/Hellström, Ingrid/Ternestedt, Britt-Marie: The modified self. Family caregivers' experiences of caring for a dying family member at home, in: Journal of Clinical Nursing 20/7-8 (2011) 1097-105.

Feichtner, Angelika/Pußwald, Bettina: Palliative Care. Unterstützung der Angehörigen, Wien: Facultas 2017.

Gallicchio, Lisa/Siddiqi, Naumann/Langenberg, Patricia/Baumgarten, Mona: Gender differences in burden and depression among informal caregivers of demented elders in the community, in: International Journal of Geriatric Psychiatry 17/2 (2002) 154-163.

George, Wolfgang/George, Ute/Bilgin, Yasar: Angehörigenintegration in der Pflege, München: Reinhardt 2003. 
Götze, Heide/Brähler, Elmar/Gansera, Lutz/Schnabel, Astrid/Köhler, Norbert: Erschöpfung und Überlastung pflegender Angehöriger von Krebspatienten in der palliativen Situation, in: PPmP - Psychotherapie - Psychosomatik - Medizinische Psychologie 65/2 (2014) 66-72.

Gomes, Barbara/Higginson, Irene J.: Factors influencing death at home in terminally ill patients with cancer. Systematic review, in: British Medical Journal 332/7540 (2006) 515-521.

Gröning, Katharina/Kunstmann, Anne-Christin/Rensing, Elisabeth: In guten wie in schlechten Tagen. Konfliktfelder in der häuslichen Pflege, Frankfurt am Main: Mabuse 2004.

Hartinger-Klein: „Pflege braucht Service und Qualität, in: https://www.ots.at/presse aussendung/OTS_20180511_OTS0024/hartinger-klein-pflege-braucht-service-un d-qualitaet [18.02.2020].

Honea, Norissa/Brintnall, Ruth Ann/Given, Barbara/Sherwood, Paula R./Colao, Deirdre B./Somers, Susan C./Northhouse, Laurel: Putting Evidence Into Practice. Nursing Assessment and Interventions to Reduce Family Caregiver Strain and Burden, in: Clinical Journal of Oncology Nursing 12/3 (2008) 507-516.

Interessengemeinschaft pflegender Angehöriger: FACTSHEET - Pflegesituation in Österreich, in: https://www.ig-pflege.at/downloads/news/2017/FACTSHEET_20 17_LOGO.pdf?m=1495009759 [18.02.2020].

Jensen, Susan/Given, Barbara: Fatigue affecting family caregivers of cancer patients, in: Support Care Cancer 1/6 (1993) 321-325.

Knighting, Katherine/O’Brien, Mary R./Roe, Brenda/Gandy, Rob/Lloyd-Williams, Mari/Nolan, Mike/Jack, Barbara: Development of the Carers' Alert Thermometer (CAT) to identify family carers struggling with caring for someone dying at home. A mixed method consensus study, in: Biomedcentral Palliative Care 14 (2015).

Kreyer, Christiane/Pleschberger, Sabine: Selbstmanagementstrategien von Familien in der Palliative Care zu Hause. Eine Metasynthese, in: Pflege 27/5 (2014) 307-324.

Kristjanson, Linda J./Aoun, Samar: Palliative Care for Families. Remembering the Hidden Patients, in: The Canadian Journal of Psychiatry 49/6 (2004) 359-365.

Kruithof, Willeke J./Post, Marcel W./Visser-Meily, Johanna M.: Measuring negative and positive caregiving experiences. A psychometric analysis of the Caregiver Strain Index Expanded, in: Clinical Rehabilitation 29/12 (2015) 1224-1233.

Kunstmann, Anne-Christin: Familiale Verbundenheit und Gerechtigkeit. Fehlende Perspektiven auf die Pflege von Angehörigen - Eine Diskursanalyse, Wiesbaden: VS Verlag für Sozialwissenschaften 2010 (= Theorie und Praxis der Diskursforschung).

Lederer, Wolfgang/Graube, Stefanie/Feichtner, Angelika/Medicus, Elisabeth: The Palliative Treatment Plan as a Bone of Contention between Attending Physicians and Nurses, in: Healthcare 3/4 (2015) 987-994. 
Nagl-Cupal, Martin/Kolland, Franz/Zartler, Ulrike/Mayer, Hanna/Bittner, Marc/ Koller, Martina/Parisot, Viktoria/Stöhr, Doreen: Angehörigenpflege in Österreich. Einsicht in die Situation pflegender Angehöriger und in die Entwicklung informeller Pflegenetzwerke, Wien: 2018.

Nagl-Cupal, Martin/Daniel, Maria/Kainbacher, Manuela/Koller, Martina/Mayer, Hanna/Hauprich, Julia: Kinder und Jugendliche als pflegende Angehörige. Einblick in die Situation Betroffener und Möglichkeiten der Unterstützung, Wien: ÖGB 2015 (= Sozialpolitische Studienreihe 19), in: http://www.studienreihe.at/c ms/Z02/Z02_2.a/1342558553555/bisherige-studien/studien-einzelband-uebersich t/band-19-kinder-und-jugendliche-als-pflegende-angehoerige [21.02.2020].

Nanni, Maria Giulia/Biancosino, Bruno/Grassi, Luigi: Pre-loss symptoms related to risk of complicated grief in caregivers of terminally ill cancer patients, in: Journal of Affective Disorders 160 (2014) 87-91.

Nielsen, Mette Kjaergaard/Neergaard, Mette Asbjoern/Jensen, Anders Bonde/Bro, Fleming/Guldin, Mai-Britt: Psychological distress, health, and socio-economic factors in caregivers of terminally ill patients. A nationwide population-based cohort study, in: Supportive Care in Cancer 24/7 (2016) 3057-3067.

Nielsen, Mette Kjaergaard /Neergaard, Mette Asbjoern/Jensen, Anders Bonde/Bro, Fleming/Guldin, Mai-Britt: Do we need to change our understanding of anticipatory grief in caregivers? A systematic review of caregiver studies during end-oflife caregiving and bereavement, in: Clinical Psychology Review 44 (2016) 7593.

Nielsen, Mette Kjaergaard/Neergaard, Mette Asbjoern/Jensen, Anders Bonde/Bro, Fleming/Guldin, Mai-Britt/Vedsted, Peter: Preloss grief in family caregivers during end-of-life cancer care. A nationwide population-based cohort study, in: Psycho-Oncology 26/12 (2017) 2048-2056.

Northfield, Sarah/Nebauer, Monica: The Caregiving Journey for Family Members of Relatives With Cancer. How Do They Cope?, in: Clinical Journal of Oncology Nursing 14/5 (2010) 567-577.

Payne, Sheila/Grande, Gunn: Towards better support for family carers. A richer understanding, in: Palliative Medicine 27/7 (2013) 579-580.

Penrod, Janice/Hupcey, Judith E./Shipley, Peggy Z./Loeb, Susan J./Baney, Brenda: A Model of Caregiving Through the End of Life. Seeking Normal, in: Western Journal of Nursing Research 34/2 (2012) 174-193.

Pochobradsky, Elisabeth/Bergmann, Franz/Brix-Samoylenko, Harald/Erfkamp, Henning/Laub, Renate: Situation pflegender Angehöriger. Endbericht (2005), in: https://broschuerenservice.sozialministerium.at/Home/Download?publicatio nId $=176$ [24.02.2020].

Sell, Stefan: Pflegende Angehörige als größter Pflegedienst der Nation - auch in Österreich. Eine Studie hat genauer hingeschaut, in: http://aktuelle-sozialpolitik. de/2018/08/19/pflegende-angehoerige-als-groesster-pflegedienst-der-nation-auch-i n-oesterreich/ [18.02.2020]. 
Seng, Boon K./Luo, Nan/Ng, Wai Yee/Lim, June/Chionh, Hui Ling/Goh, Jenny/ Yap, Phillip: Validity and reliability of the Zarit Burden Interview in assessing caregiving burden, in: Annals of the Academy of Medicine 39/10 (2010) 758763.

Stajduhar, Kelli/Davies, Betty: Variations in and factors influencing family members' decisions for palliative home care, in: Palliative Medicine 19/1 (2005) 2132.

Stajduhar, Kelli/Funk, Laura/Toye, Christine/Grande, Ge/Aoun, Samar/Todd, Chris J.: Part 1: Home-based family caregiving at the end of life. A comprehensive review of published quantitative research (1998-2008), in: Palliative Medicine 24/6 (2010) 573-593.

Stajduhar, Kelli: Burdens of family caregiving at the end of life, in: Clinical and Investigative Medicine 36/3 (2013) E121-E126.

Statistik Austria: Statistik der natürlichen Bevölkerungsbewegung, in: http://www.s tatistik.at/webde/statistiken/menschen_und_gesellschaft/bevoelkerung/gestorbe ne/index.html [18.05.2018].

Stenberg, Una/Ruland, Cornelia M./Olsson, Mariann/Ekstedt, Mirjam: To Live Close to a Person With Cancer. Experiences of Family Caregivers, in: Social Work in Health Care 51 (2012) 909-926.

Wright, Lorraine M./Leahey, Maureen: Calgary Family Intervention Model. One way to think about change, in: Journal of Marital and Family Therapy 20 (1994) 381-395.

Zalenski, Robert J./Raspa, Richard: Maslow's Hierarchy of Needs. A Framework for Achieving Human Potential, in: Hospice.Journal of Palliative Medicine 9/5 (2006) 1120-1127.

Zarit, Steven H./Todd, Pamela A./Zarit, Judy M.: Subjective burden of husbands and wives as caregivers. A longitudinal study, in: Gerontologist 26/3 (1986) 260266. 